\title{
Optical CCD photometry of the microquasar LS 5039
}

\author{
J. Martí ${ }^{1}$, P. Luque-Escamilla ${ }^{2}$, J. L. Garrido ${ }^{1}$, J. M. Paredes ${ }^{3}$, and R. Zamanov ${ }^{4}$ \\ ${ }^{1}$ Departamento de Física, Escuela Politécnica Superior, Universidad de Jaén, Virgen de la Cabeza 2, 23071 Jaén, Spain \\ e-mail: jlg@ujaen.es \\ 2 Departamento de Ingeniería Mecánica y Minera, Escuela Universitaria Politécnica de Linares, Universidad de Jaén, \\ C/ Alfonso X el Sabio 28, 23700 Linares, Spain \\ e-mail: peter@ujaen.es \\ 3 Departament d'Astronomia i Meteorologia, Universitat de Barcelona, Av. Diagonal 647, 08028 Barcelona, Spain \\ e-mail: jmparedes@ub.edu \\ 4 Astrophysics Research Institute, Liverpool John Moores University, Twelve Quays House, Birkenhead, CH41 1LD, UK \\ e-mail: rz@astro.livjm.ac.uk
}

Received 16 December 2003 / Accepted 15 January 2004

\begin{abstract}
We report optical CCD observations of the microquasar LS 5039. Its photometric behaviour is studied over different orbital periods with a time separation of three years. We do not detect evidences of an ellipsoidal modulation above the $\pm 0.01 \mathrm{mag}$ level, thus confirming the previous results reported by Clark et al. (2001). The light curve of LS 5039 has also been explored with a time resolution of $30 \mathrm{~s}$ but the source also appears to be stable on such shorter time scales. Possible scenarios consistent with the non-detection of optical variability are considered.
\end{abstract}

Key words. stars: individual: LS 5039 (RX J1826.2-1450) - X-rays: binaries - radio continuum: stars

\section{Introduction}

LS 5039/RX J1826.2-1450 is a high mass X-ray binary (HMXB) with relativistic jets belonging to the class of microquasar systems. Attention to this object was first called by Motch et al. (1997) who proposed it as a HMXB candidate based on ROSAT data. Subsequently, Martí et al. (1998) reported the discovery of non-thermal radio emission associated with it being persistent but moderately variable. Paredes et al. (2000) were able to resolve the radio emission into collimated jets at milli-arcsecond angular scales, thus establishing a microquasar classification for this object. These authors also pointed out the likely association between LS 5039 and the unidentified gamma-ray source 3EG J1824-1514.

The confirmation of the binary nature of LS 5039 came from Doppler shifts in the optical spectrum which revealed a significantly eccentric binary system (McSwain et al. 2001). The orbital period and spectroscopic classification have been recently improved by McSwain et al. (2004), who report a value of $P_{\text {orb }}=4.4267 \pm 0.0005 \mathrm{~d}$ and a spectral type of ON6.5V((f)) corresponding to a nitrogen rich star (see also consistent results in Casares et al. 2004). The small value of the mass function derived from the radial velocity curve points to a neutron star as the compact companion of the system.

Send offprint requests to: J. Martí,

e-mail: jmarti@ujaen.es
At optical wavelengths, LS 5039 displays little variability on time scales from months to years around $V=11.2$ (Clark et al. 2001). In the infrared, the same authors have reported significant variability $(\sim 0.4 \mathrm{mag})$ in the $J H K$ bands over a time scale of years. Its origin is however not yet clear. Another interesting property of LS 5039 is its runaway velocity escaping from the Galactic Plane at more than $100 \mathrm{~km} \mathrm{~s}^{-1}$ relative to its regional Local Standard of Rest (Ribó et al. 2002). A recent account of the LS 5039 X-ray properties can be found in Reig et al. (2003).

In this paper, we re-visit the photometric behavior of LS 5039 focusing our attention on its variability properties. On time scales comparable with the orbital period, our goal was to look for evidence of any possible orbital modulation. Periodic ellipsoidal variations are a common phenomenon among HMXBs due to tidal distorsions of the optical star, provided that the optical companion fills its Roche lobe. On much shorter time scales, we were also interested to look for possible fast variability which could be related to accretion/ejection events in this microquasar.

Finally, we mention that our observing dates in 2003 were arranged to provide simultaneous optical monitoring during a series of pointings with the Rossi X-ray Timing Explorer (RXTE) satellite (to be reported elsewhere). 


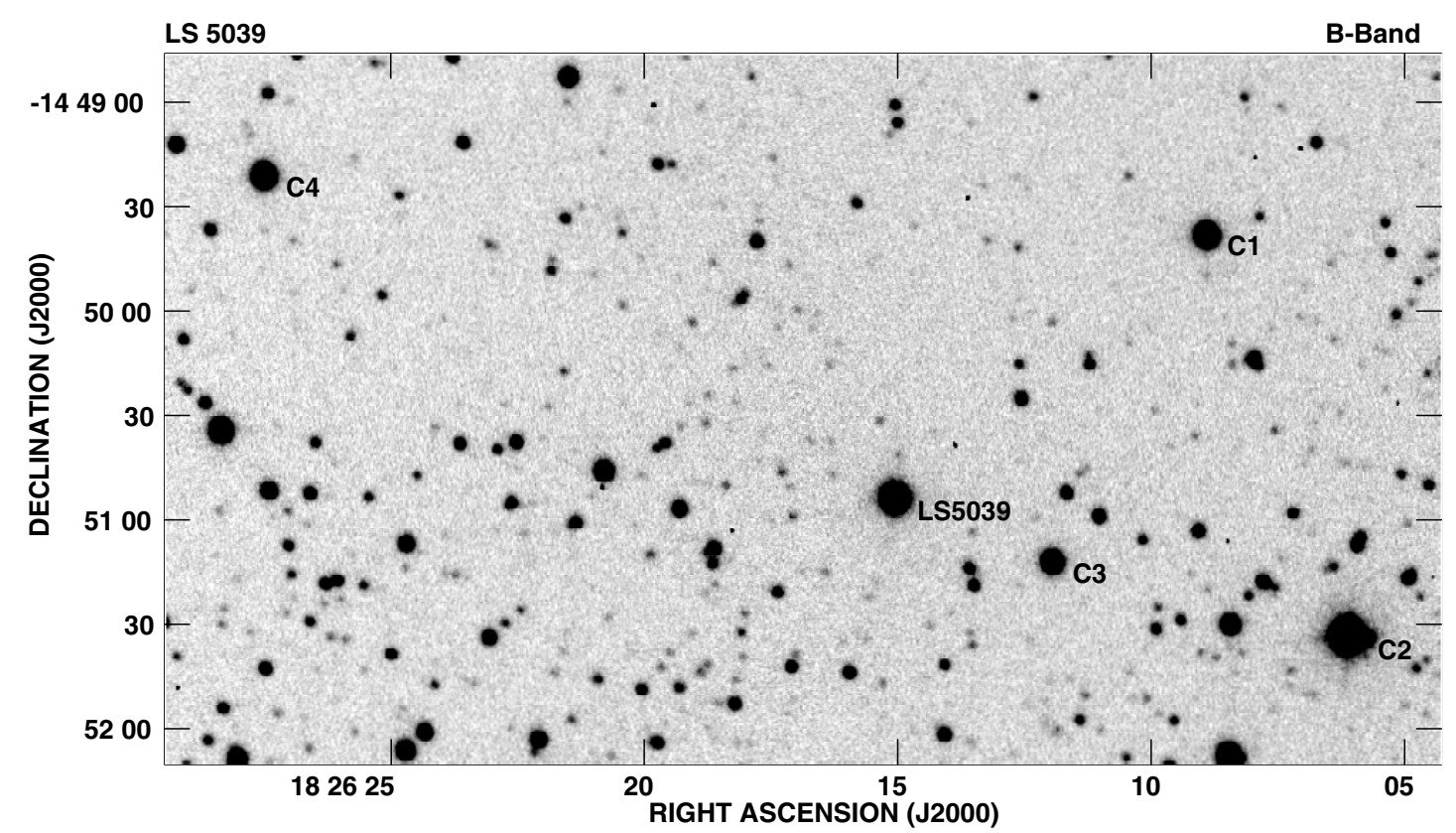

Fig. 1. Finding chart of LS 5039 showing the optical counterpart of the microquasar and the comparison stars selected in the field. This image is one of the CCD frames taken with the $1.52 \mathrm{~m}$ OAN telescope under good seeing conditions.

Table 1. Average magnitudes of comparison and target stars.

\begin{tabular}{ccccc}
\hline \hline Star & $B$ & $V$ & $R$ & $I$ \\
& $N_{\text {obs }}=35$ & $N_{\text {obs }}=22$ & $N_{\text {obs }}=3$ & $N_{\text {obs }}=2$ \\
\hline C1 & 13.26 & 12.42 & 11.92 & 11.46 \\
C2 & 11.24 & 9.94 & 9.22 & 8.56 \\
C3 & 13.92 & 12.86 & 12.12 & 11.38 \\
C4 & 13.26 & 11.69 & 10.79 & 9.99 \\
LS 5039 & 12.24 & 11.23 & 10.53 & 9.83 \\
\hline
\end{tabular}

\section{Observations and results}

Photometric observations of LS 5039 were carried out at different observatories and we present them below. In all cases, the CCD images were acquired using a wheel of standard $B V R I$ Johnson-Kron-Cousins filters. The CCD frames were reduced using the IRAF software package, including bias subtraction and flat fielding with sky flats. Aperture photometry was later performed using the PHOT task of IRAF.

\subsection{OAN observations}

These observations were carried out with the $1.52 \mathrm{~m}$ telescope of the Observatorio Astronómico Nacional (OAN) in Calar Alto (Spain) from 2003 July 1 to 6 in coordination with RXTE pointings. The post-focus instrumentation consisted of a CCD camera with a TK1024AB chip $\left(1024 \times 1024\right.$ pixel $\left.^{2}\right)$ and cooled at about $-110{ }^{\circ} \mathrm{C}$ with liquid $\mathrm{N}_{2}$. The corresponding field of view was $6.9 \times 6.9 \mathrm{arcmin}^{2}$, with a pixel scale of 0.' 40 pixel $^{-1}$. The absolute calibration of the photometry was performed by observing several standard stars from Landolt (1992) over a wide range of air masses during the only acceptable photometric night (2003 July 5th). The seeing conditions ranged from $\sim 1.5^{\prime \prime}$ to $\sim 3^{\prime \prime}$.
Four isolated stars in the LS 5039 field of view were chosen as possible comparison stars for differential photometry. They are labelled as C1, C2, C3 and C4 in Fig. 1. Their average magnitudes are collected in Table 1 together with those of LS 5039 and the total number of observations $\left(N_{\text {obs }}\right)$. Errors in the standard photometry of this table are estimated to range from \pm 0.04 mag in $V$ to \pm 0.09 in $I$. The listed values are consistent with those previously given in Martí et al. (1998) within $0.1 \mathrm{mag}$.

The final photometry of LS 5039 is presented in Fig. 2 for the $V$ and $B$ bands, which are the ones most intensively observed.

The plotted values are based on the $\mathrm{C} 3$ star magnitude according to Table 1. This is the comparison star closer to LS 5039 and also has practically the same $B-V$ colour as the microquasar. The magnitude differences between $\mathrm{C} 3$ and $\mathrm{C} 4$, used as a check star, were always consistent within \pm 0.01 mag or less.

\subsection{CAHA observations}

LS 5039 was observed with the 1.23 m telescope of the Centro Astronómico Hispano Alemán (CAHA), also located in Calar Alto (Spain), during the dates between 2000 June 22 and 28. The telescope was equipped with a SITe\#2b_17 chip $(2048 \times$ 2048 pixel $^{2}$ ) and the CCD frames were processed similarly as the OAN observations. The detector was also cooled with $\mathrm{N}_{2}$ at $-96{ }^{\circ} \mathrm{C}$. Only differential photometry was performed with the adopted magnitudes for the comparison stars being the same as in Table 1. The seeing conditions were in a range comparable with the OAN run. At all times, the magnitude differences between $\mathrm{C} 3$ and $\mathrm{C} 4$ remained also constant and consistent with the OAN values within $\pm 0.01 \mathrm{mag}$. The CAHA individual data 


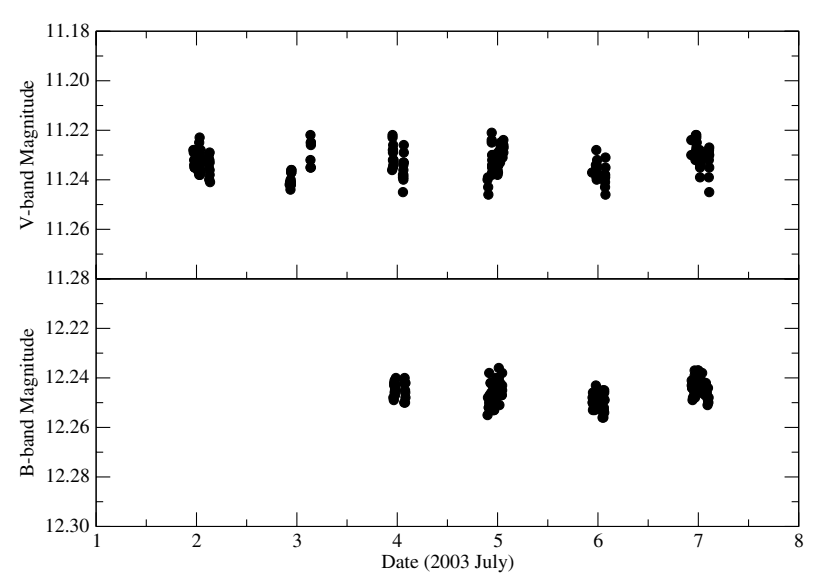

Fig. 2. Results of OAN differential photometry of LS 5039 during 2003 July using the comparison star C3 (see Table 1). Each data point corresponds a single CCD frame. The top and bottom panel contain the $V$ and $B$-band photometry, respectively.

points are shown in Fig. 4 together with the OAN observations folded with the orbital period.

On the other hand, during the night of June 22/23 we devoted several hours of telescope time to sample the LS 5039 light curve with sub-minute time resolution. We used for this purpose a small window of the CCD chip $\left(400 \times 400\right.$ pixel $\left.^{2}\right)$ thus providing high speed photometry in the $V$-band. In this way, LS 5039 was monitored with time resolution of $30 \mathrm{~s}$ for nearly three hours. The resulting light curve is displayed in Fig. 3.

\section{Discussion}

LS 5039 is known to be optically very stable over the years within $0.1 \mathrm{mag}$ (see e.g. the historical photometry collected in Clark et al. 2001). Only in the infrared there seems to be significant variability of unclear origin as mentioned in the introduction.

Here, we are mainly interested on time scales comparable with the orbital period of the binary $(4.4267 \mathrm{~d})$. In Fig. 2 we can see that LS 5039 displayed no photometric variability above \pm 0.01 mag throughout the six consecutive nights (1.3 orbital cycles) of the OAN run. Such stability becomes more evident when folding all photometric data points (OAN plus CAHA) on the orbital period. The photometry as a function of orbital phase is displayed in Fig. 4 for both runs.

The folded optical light curve in Fig. 4 is essentially flat with no evidence of ellipsoidal modulation superimposed on it within our photometric accuracy. It is important to stress here that the points plotted in phase come from runs separated by three years in time. If a modulation was present at the time of our 2000 and 2003 observations, its amplitude has to be smaller than $\pm 0.01 \mathrm{mag}$. This fact is in good agreement with the previous results on medium term variability reported by Clark et al. (2001), based on 21 nights of optical monitoring during 2000. Hints of day to day variability, at a few 0.01 mag level, were present in preliminary observations with the OAN telescope (Martí et al. 1998). Nevertheless, such variations could not be confirmed with our new OAN and CAHA data.

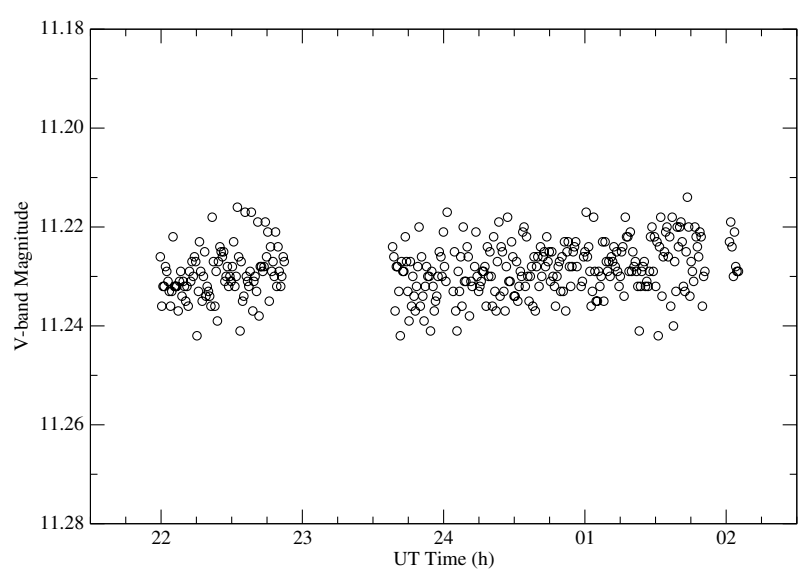

Fig. 3. Light curve of LS 5039 as observed with the 1.23 m CAHA telescope during the night of 22/23 June 2000. The time resolution of this $V$-band photometry is $30 \mathrm{~s}$. The same comparison star $\mathrm{C} 3$ of the OAN observations (see Table 1) has been used in order to set the magnitude scale.

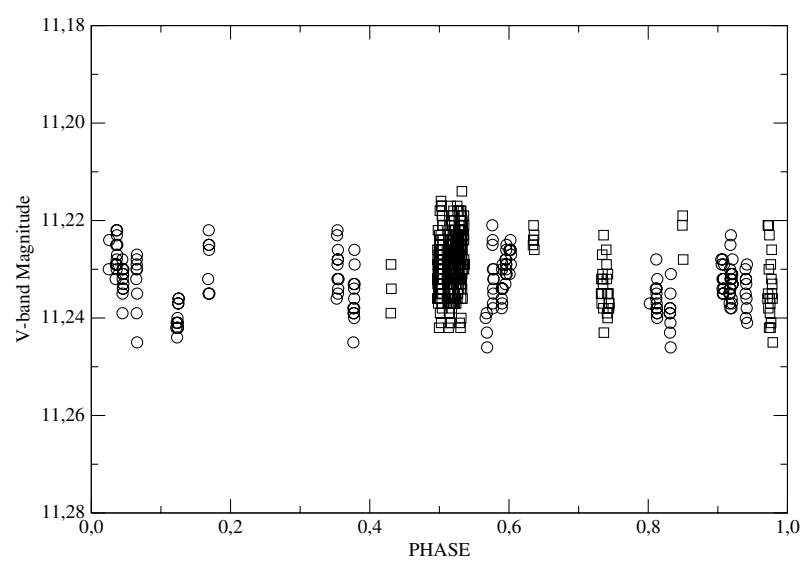

Fig. 4. OAN (circles) and CAHA (squares) photometry of LS 5039 folded together on the orbital period. Only $V$-band data with a better time coverage than other bands are included. Phase zero has been set at MJD 52755.99 according to the McSwain et al. (2004) ephemeris.

On the other hand, no reliable evidence for intra-night variability could be found at the few hundredths of magnitude level as Clark et al. (2001) mention in their paper. This short term variability, in time scales of hours, does not seem to be always present as clearly indicated by our almost flat high speed photometry (see Fig. 3). We cannot exclude here that any short term fluctuations are a phase dependent phenomenon given the significant eccentricity of the binary system.

Taking together both the Clark et al. (2001) results and ours, expanding over a time baseline of several years, it seems now well established that a significant orbital modulation does not exist in the optical light curve of LS 5039. The absence of variability with the orbital period could be consistent with one of the two following scenarios.

In a system with Roche lobe overflow, an orbital ellipsoidal modulation from a tidally distorted star would be expected except for a very low inclination of the orbital plane. The best upper limits on inclination currently available come from the absence of X-ray eclipses and they are not particularly strong 
to suggest an almost pole-on orbital plane. Reig et al. (2003) derive $i \leq 66^{\circ}$ and $i \leq 68^{\circ}$ for a $1.4 M_{\odot}$ and $9.0 M_{\odot}$ primary companion, respectively. Thus, this interpretation does not seem very likely because it requires a fine tuning of the inclination parameter.

A second, more plausible scenario is LS 5039 being a wind powered X-ray binary (Martí et al. 1998; McSwain et al. 2001, 2002) with the donor star not filling its Roche lobe. This alternative interpretation is more consistent with the low X-ray luminosity of the system and the nonappearance of $\mathrm{H} \alpha$ emission in its spectrum. Moreover, it does not impose strong constrains on the possible inclinations allowed. The value $i \sim 50^{\circ}$ suggested by McSwain et al. (2004) from wind accretion models would be perfectly conceivable here.

The stability in the optical flux is in contrast with the occasional variability of LS 5039 when observed in optical polarized light (Combi et al. 2003), in time scales of hours. Such variations, if confirmed, could perhaps be attributed to local density enhancements in the stellar wind of the ON6.5V((f)). Extrapolating the synchrotron radio spectrum of $\operatorname{LS} 5039\left(S_{v}=\right.$ $(52 \pm 1) \mathrm{mJy}(v / 1 \mathrm{GHz})^{-0.46 \pm 0.01}$, Martí et al. 1998) to optical wavelengths gives indeed a negligible contribution compared with the optical star (of order $\sim 10^{-4}$ ). Thus variability in the optical domain is not likely expected from the jets and we should go to infrared, or longer wavelengths, before the non-thermal component in LS 5039 begins to be noticeable in its photometric behavior. Our observations also suggest that the possible accretion disk in LS 5039 does not affect significantly its optical light curve.

Further observations, specially at infrared wavelengths, will be required to fully characterize the photometric properties of LS 5039. Open issues yet to be solved are the origin and possible connection of eventual intra-night fluctuations at the few 0.01 mag level, polarization changes and long-term infrared variability.

\section{Conclusions}

We have reported new BVRI photometry of the microquasar system LS 5039. Our main conclusions can be summarized as follows:

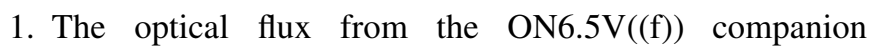
does not display evidence for an ellipsoidal light curve due to a tidally distorted star. The observed stability was at the \pm 0.01 mag level during different orbital periods separated by several years.

2. Our results are in good agreement with those from Clark et al. (2001) and reinforce the idea that LS 5039 is a wind powered X-ray binary where the optical star is far from filling its Roche lobe.

3. Our data, with time resolution ranging from days to minutes, also suggests that the non-thermal, and moderately variable synchrotron emission from the relativistic jet does not have detectable effects at optical wavelengths where the constant luminosity of the ON6.5V((f)) star dominates. The absence of optically detectable effects likely applies to the accretion disk as well.

Acknowledgements. Visiting Astronomer, German-Spanish Astronomical Centre, Calar Alto, operated by the Max-PlanckInstitute for Astronomy, Heidelberg, jointly with the Spanish National Commission for Astronomy. J.M. and J.M.P. acknowledge partial support by DGI of the Ministerio de Ciencia y Tecnología (Spain) under grant AYA2001-3092, as well as partial support by the European Regional Development Fund (ERDF/FEDER). J.M., P.L.E. and J.L.G. are also supported by the Junta de Andalucía (Spain) under project FQM322. We also thank J. Casares (IAC) for useful comments and suggestions in the early stages of this work.

\section{References}

Casares, J., Ribó, M., Paredes, J. M., \& Martí, J. 2004, in preparation Clark, J. S., Reig, P., Goodwin, S. P., et al. 2001, A\&A, 376, 476

Combi, J., et al. 2003, private communication

Landolt, A. U. 1992, AJ, 104, 340

Martí, J., Paredes, J. M., \& Ribó, M. 1998, A\&A, 338, L71

McSwain, M. V., Gies, D. R., Riddle, R. L., Wang, Z., \& Wingert, D. W. 2001, ApJ, 558, L43

McSwain, M. V., Gies, D. R. 2002, ApJ, 568, L27

McSwain, M. V., Gies, D. R., Huang, W. et al. 2004, ApJ, 600, 927

Motch, C., Haberl, F., Dennerl, K., Pakull, M., \& Janot-Pacheco, E. 1997, A\&A, 323, 853

Paredes, J. M., Martí, J., Ribó, M., \& Massi, M. 2000, Science, 288, 2340

Paredes, J. M., Ribó, M., Ros, E., Martí, J., \& Massi, M. 2002, A\&A, 393, L99

Reig, P., Ribó, M., Paredes, J. M., \& Martí, J. 2003, A\&A, 405, 285

Ribó, M., Paredes, J. M., Romero, G. E., et al. 2002, A\&A, 384, 954 